Reprod. Nutr. Dévelop., 1981, 21 (2), 257-264.

\title{
Expression du génome de la cellule séricigène de Bombyx mori au dernier stade larvaire
}

\author{
par P. COUBLE, Annie GAREL, J.-C. PRUDHOMME \\ avec la collaboration technique de Mme M.-A. GUÉRIN \\ Département de Biologie générale et appliquée, \\ Laboratoire associé au C.N.R.S. no 92, Université Claude-Bernard Lyon-I \\ 43, Boulevard du 11-Novembre-1918, 69622 Villeurbanne Cedex.
}

Summary. Gene expression in Bombyx mori silkgland cells during the last larval instar.

Poly (A)-containing RNA was isolated from the posterior silkgland of Bombyx mori just after the fourth molt, when the gland is not producing fibroin (stage $V_{0}$ ), and at the middle of the fifth larval instar, when the fibroin is massively synthesized $\left(\right.$ stage $V_{6}$ ). The hybridization kinetics of these mRNA with their complementary DNA (cDNA) were remarkably different. The mRNA from stage $V_{0}$ consisted of two abundance classes, comprising 38 and 2915 different species, respectively. At stage $V_{6}$, fibroin mRNA (FmRNA) was separated from the rest of the poly (A)-containing RNA ( $F^{-}$mRNA) by centrifugation on sucrose gradients, and both preparations were analyzed separately. The kinetic complexity of FmRNA was very low as compared to its actual size. This result agrees with the existence of a short repetitive sequence accounting for 60 p. 100 of the molecule. Stage $V_{6} F^{-}$mRNA was resolved into four classes containing 1, 20, 319 and about 2600 species, respectively.

We carried out cross-hybridization with each of the two cDNA classes from stage $V_{0}$ and stage $V_{6} F^{-}$mRNA. They demonstrated that almost all the sequences present at stage $V_{0}$ were also present at stage $V_{6}$, but that their abundance class distribution was modified.

Our data show that the specialization of the silkgland cell for fibroin production is characterized by massive accumulation of FmRNA and another unknown species, and that stage variations of $m$ RNA populations are related to relative quantitative variations rather than to qualitative ones.

\section{Introduction.}

Au dernier stade larvaire, l'activité des cellules de la partie postérieure de la glande séricigène du Ver à soie s'oriente vers la production massive de la fibroïne qui représente alors environ 80 p. 100 de la synthèse protéique. A ce moment, la cellule séricigène présente des caractéristiques histologiques ef biochimiques spécifiques. Celles-ci se traduisent par un développement intense des organites impliqués dans la synthèse (réticulum endoplasmique rugueux, RER) ef le transport (appareil de Golgi) de la fibroïne. De plus, il y a accumulation de l'ARN messager de la fibroïne (mRNAF) (revue dans Prudhomme ef Couble, 1979), ainsi que des macromolécules qui assurent le métabolisme des acides aminés et le fonctionnement de l'appareil de tra- 
duction. Chez l'animal en sortie de dernière mue larvaire, environ 6 jours plus tôt, la situation est bien différente. La cellule a subi au cours de la mue d'intenses remaniements cellulaires qui ont abouti à la disparition quasi complète du RER et à la régression de l'appareil de Golgi (Tashiro et al., 1968). Par ailleurs, le mRNAF a été détruit presque totalement (Suzuki et Suzuki, 1974) et la cellule ne synthétise pas de fibroïne.

Il est ainsi possible de définir deux états fonctionnels bien différents : à la sortie de mue (stade noté $V_{0}$ ) la cellule n'exprime pas sa fonction spécifique alors qu'au milieu du dernier stade larvaire (stade $V_{6}$ ) sa spécialisation est maximale.

Si l'évolution quantitative du mRNAF entre ces deux états est bien connue (revue dans Suzuki, 1975 et 1977), on ne connaît rien des variations des pools des autres mRNA, variations qui doivent a priori, rendre compte des modifications décrites cidessus qui accompagnent la spécialisation de la cellule dans la production de la fibroïne. C'est dans le but de définir ces variations et de rechercher s'il existe une «famille » de gènes impliqués dans la spécialisation cellulaire que nous avons entrepris l'étude des mRNA de la cellule séricigène aux deux stades précédemment définis (Couble, Garel et Prudhomme, 1981).

\section{Matériel et méthodes.}

Le détail des techniques mises en jeu, décrit par ailleurs (Couble, Garel ef Prudhomme, 1981), est résumé ci-dessous.

Les Vers à soie utilisés sont des hybrides des races européennes 200 et 300 . lls sont élevés au laboratoire à $22^{\circ} \mathrm{C}$ sur feuilles de mûrier.

Le RNA total est extrait selon une adaptation de la technique de Greene et al. (1975). Elle consiste en une extraction phénolique de l'homogénat de glande réalisé dans le tampon suivant : $30 \mathrm{mM}$ Tris-Cl ( $\mathrm{pH} 7,5), 100 \mathrm{mM} \mathrm{NaCl}, 1 \mathrm{mM}$ EDTA, confenant 0,5 p. 100 de dodécyl-sulfate de sodium (SDS). Les mRNA polyadénylés sont obtenus par chromatographie sur colonne d'oligo (dT)-cellulose selon Aviv ef Leder (1972), le RNA total étant chauffé pendant 2 min à $65^{\circ} \mathrm{C}$ avant dépôt sur la colonne. Le mRNAF est purifié par centrifugation sur gradient de saccharose, il sédimente à $47 \mathrm{~S}$ alors que la masse des autres mRNA (fraction notée $\mathrm{F}^{-}$) sédimente en dessous de $28 \mathrm{~S}$ avec un mode à $15 \mathrm{~S}$.

Les DNA complémentaires (cDNA) des mRNA sont obtenus par action de la « reverse transcriptase » dans les conditions d'Axel, Feigelson et Schütz (1976) avec les modifications développées par Myers et Spiegelman (1978). Le rendement de la réaction est de l'ordre de 11 p. 100 pour la fraction $F^{-}$, il est 8 fois moindre pour le FmRNA. L'hybridation cDNA-mRNA est effectuée selon Axel, Feigelson et Schüłz (1976) dans un volume final de $100 \mu l$ contenant $1 \mathrm{mM}$ Tris- $\mathrm{Cl}(\mathrm{pH} 7,5), 0,2 \mathrm{mM}$ EDTA, $0,4 \mathrm{M} \mathrm{NaCl}$ et 0,1 p. 100 SDS. La concentration en cDNA est de 2-3 $\mathrm{ng} / \mathrm{ml}$ et le rapport RNA/DNA n'est jamais inférieur à 20. Le comptage des hybrides est effectué après action de la nucléase $\mathrm{S1}$ d'Aspergillus oryzae à la concentration de 1000 unités $/ \mathrm{ml}$. La complexité des populations de mRNA a été calculée à partir des cinétiques d'hybridation en utilisant comme standard la complexité du mRNA d'ovalbumine ( 2000 nucléotides) dont le $R_{0} \dagger 1 / 2$, dans nos conditions d'hybridation, est de $4.10^{-4}$ (Hynes et al., 1977). 
Les classes d'abondance des CDNA sont fractionnées par hybridation jusqu'à la valeur de $R_{0} \dagger$ choisie et les hybrides sont séparés des CDNA non hybridés par chromatographie sur colonne d'hydroxyapatite selon Hastie et Bishop (1976). L'analyse de la słabilité thermique des hybrides est effectuée également par chromatographie sur hydroxyapatite selon le protocole de Kemp (1975).

\section{Résultats.}

1. Analyse de la complexité des mRNA au stade $V_{6}$. - Etant donné que le mRNAF n'est pas copié par la « reverse transcriptase» avec le même rendement que les autres mRNA, ce qui est probablement dô à sa grande taille (16000 nucléotides), nous avons analysé séparément la complexité du mRNAF et celle de la fraction débarrassée de ce messager (fraction $\mathrm{F}^{-}$).

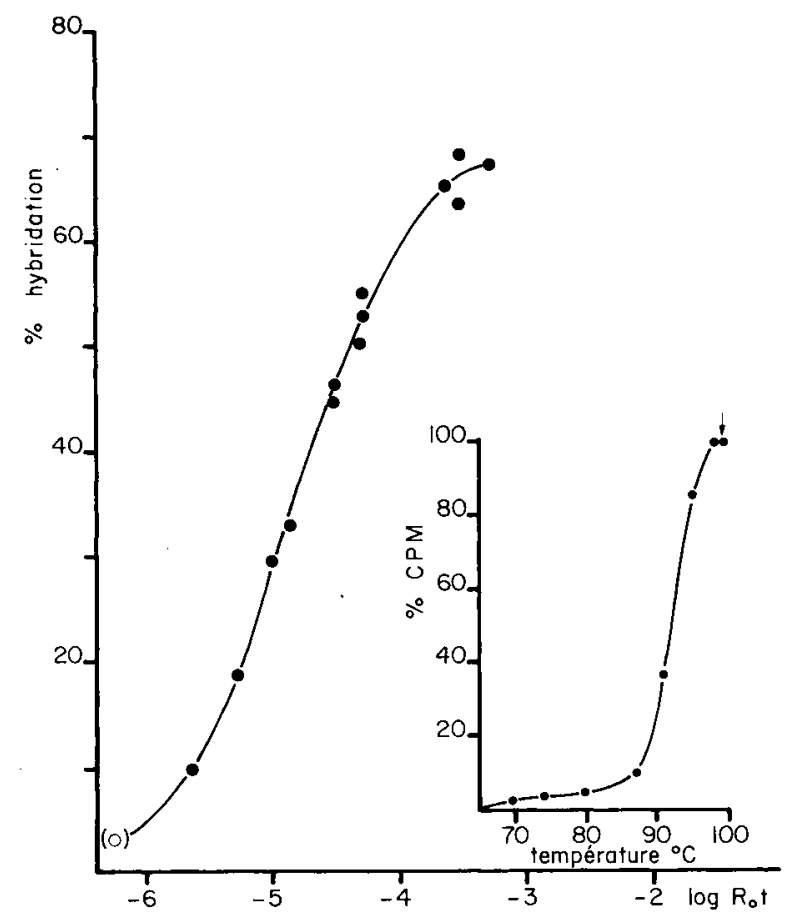

FIG. 1. - Hybridation du mRNAF avec le DNA complémentaire.

Le pourcentage d'hybridation est représenté en fonction du logarithme de $R_{0} t$, produit de la concentration du mRNA (en moles de nucléotides/I) par le temps en sec. L'encart représente la courbe de dénaturation thermique de l'hybride $\left(T_{m}=92,5^{\circ} \mathrm{C}\right)$.

La courbe d'hybridation du mRNAF avec son cDNA (fig. 1) montre une grande vitesse de réaction, le $R_{0} \dagger 1 / 2$ de $2.10^{-5}$ correspondant à une faible complexité, de l'ordre de 100 nucléotides, très inférieure à la taille du mRNAF. Cette remarquable vitesse d'hybridation est probablement due à l'existence d'une brève séquence répétitive à l'intérieur de la molécule (Suzuki et Brown, 1972). Une complexité cinétique 
du même ordre - 65 nucléotides - a été obtenue par Morrow ef al. (1977) dans des expériences de renaturation du gène de la fibroïne.

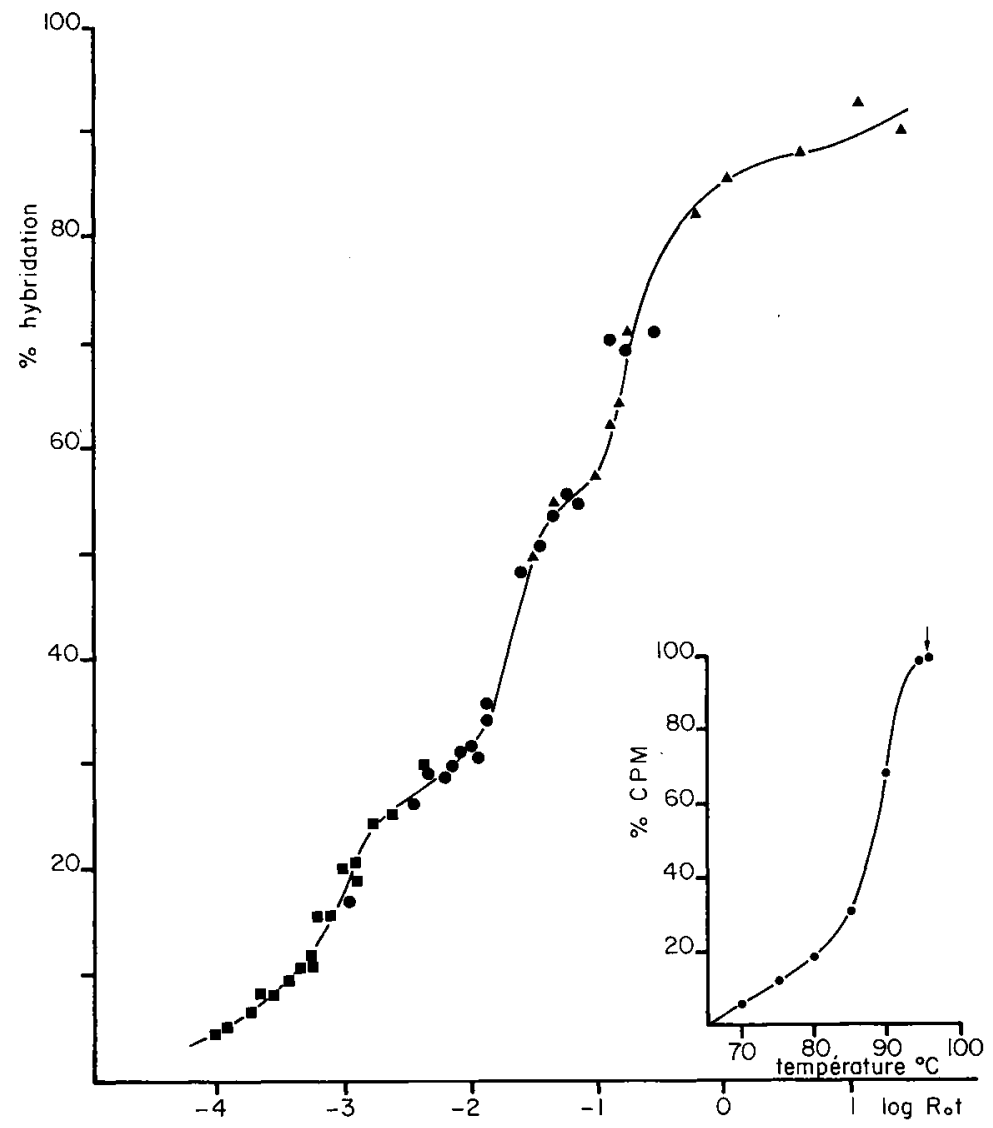

FIG. 2. - Hybridation du mRNA $V_{6} F^{-}$ovec le DNA complémentaire.

Même représentation que la figure 1 . Les symboles $(\bullet, \bullet, \Lambda)$ correspondent d̀ différentes concentrations initiales de mRNA. La $T_{m}$ est de $88^{\circ} \mathrm{C}$.

La cinétique d'hybridation de la fraction $\mathrm{F}^{-}$avec son cDNA s'étend sur 5 ordres de grandeur, ce qui reflète l'extrême hétérogénéité de cette population (fig. 2). Trois classes d'abondance sont apparentes, elles contiennent respectivement 1, 20 et 319 espèces différentes. En fait, il existe une quatrième classe contenant environ 2600 espèces qui ne représente qu'un faible pourcentage de cDNA ef qu'on ne peut défecter que par hybridation hétérologue (voir plus loin).

2. Analyse de la complexité des mRNA au stade $V_{0}$. - La cinétique d'hybridation obtenue (fig. 3) révèle l'existence d'une population de mRNA moins hétérogène qu'au stade $V_{6}$ puisque finalement deux classes d'abondance contenant respectivement 38 ef 2915 espèces différentes peuvent être mises en évidence. 


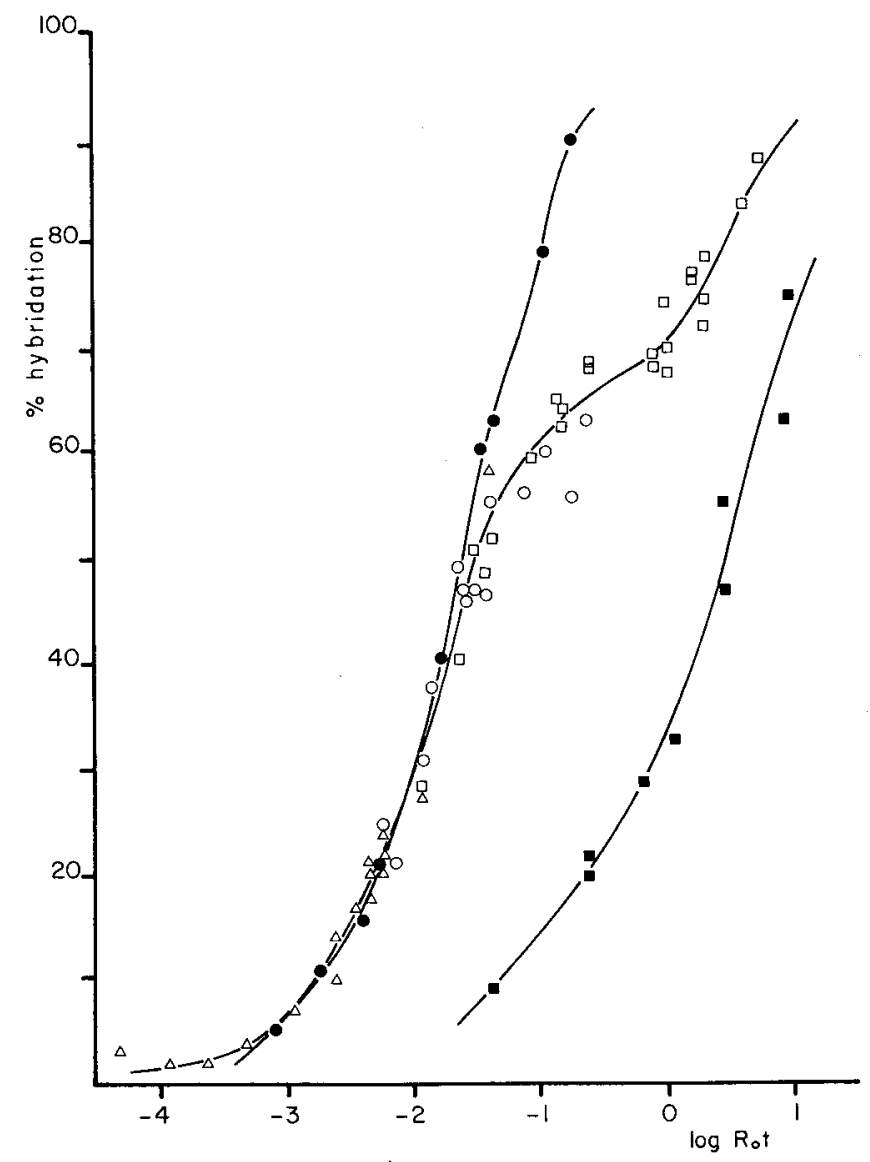

FIG. 3. - Hybridation du mRNA $V_{0}$ avec le DNA complémentaire.

Les symboles $(\Delta, \circ, \square)$ correspondent à différentes concentrations initiales. Les hybridations du mRNA $V_{0}$ avec les cDNA de chacune des deux classes, abondante et rare, sont respectivement représentées par les symboles $(\bullet)$ et $(\boldsymbol{\bullet})$.

3. Hybridations hétérologues CDNA $V_{0}$-mRNA $V_{6}$. - Les CDNA des deux classes d'abondance du stade $V_{0}$ ont été isolés ef soumis à l'hybridation avec le mRNA Fdu stade $V_{6}$ (fig. 4). Le cDNA de la classe abondante (à savoir 38 espèces) s'hybride à 95 p. 100 avec le mRNA $F^{-} V_{6}$, c'est-à-dire que la quasi-totalité des espèces de cette classe est présente également au stade $V_{6}$. Elles s'y distribuent en deux classes représentant respectivement 25 espèces et 13 espèces qui s'hybrident à des $R_{0} \ddagger 1 / 2$ correspondant aux classes 2 et 3 du stade $V_{6}$.

Le cDNA de la classe rare du stade $V_{0}$ s'hybride à $80 \mathrm{p}$. 100 avec une cinétique également biphasique. La première classe - environ 10 p. 100 soit 290 espèces s'hybride au $R_{0}+1 / 2$ de la classe 3 . La seconde classe contenant au moins 2600 espèces s'hybride à des valeurs de $R_{0}$ t supérieures à celles qui sont observées dans l'hybridation homologue $V_{6}$. C'est la mise en évidence de l'existence de la quatrième classe du stade $V_{6}$. 


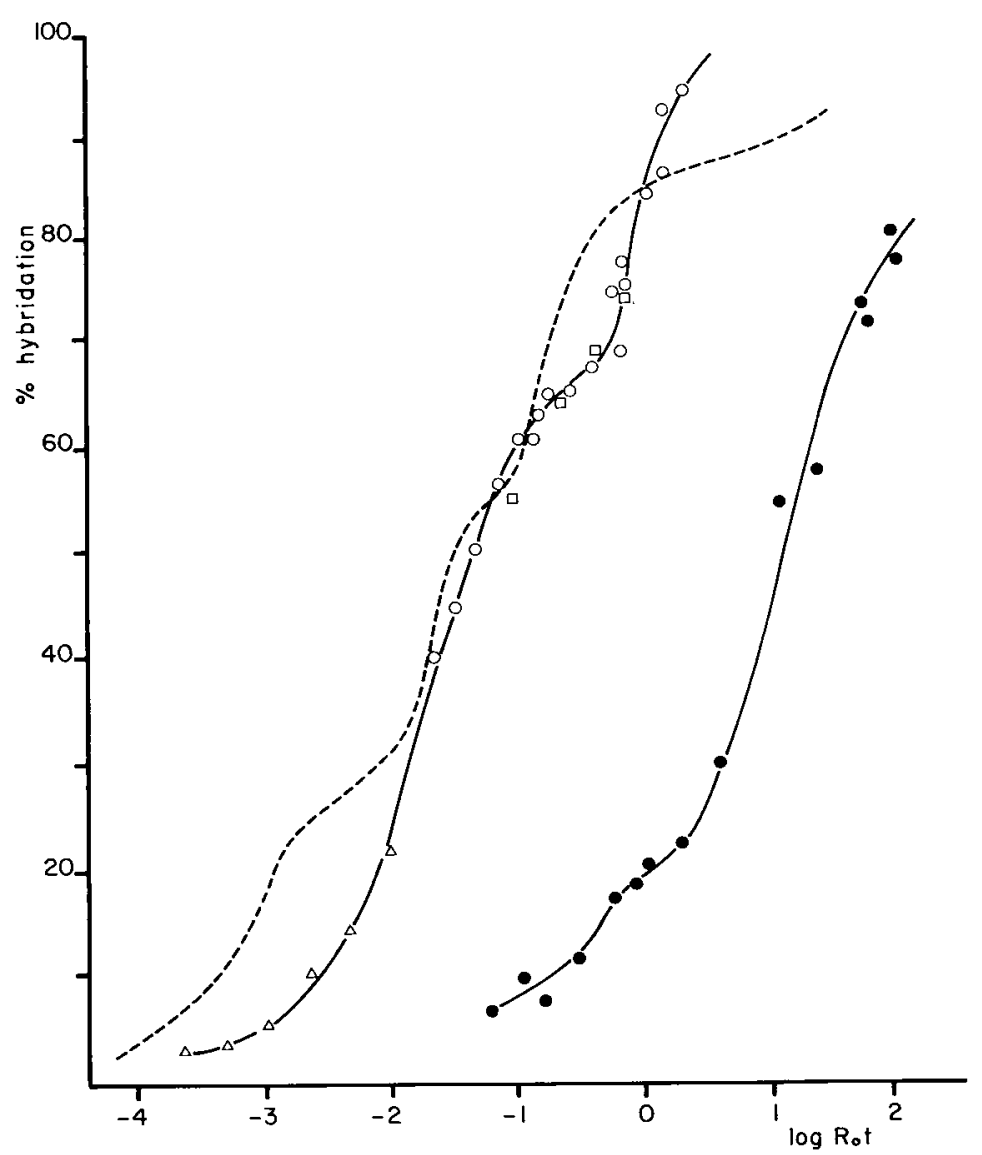

FIG. 4. - Hybridations croisées du mRNA $V_{6} F^{-}$avec les CDNA des deux closses du stade $V_{0}$. Le mRNA $V_{6} F^{-}$a été hybridé avec le cDNA de la classe abondante $(\Delta, \square, 0)$ et celui de la classe rare (๑) du stade $V_{0}$. La courbe en pointillé représente l'hybridation homologue $V_{6}$ de la figure 2 .

4. Présence du mRNAF ef de la classe 1 du stade $V_{6}$ au stade $V_{0^{\circ}}$ - Le cDNA du mRNA de fibroīne a été hybridé avec le mRNA $V_{0}$. La valeur de $R_{0} \uparrow 1 / 2$ obtenue correspond à la présence de ce mRNA dans la classe rare du stade $V_{0}$.

Le cDNA de la classe 1 du stade $V_{6}$ a été isolé puis hybridé avec le mRNA $V_{0}$. On constate alors qu'il s'hybride avec le $R_{0} t 1 / 2$ caractéristique de la classe abondante du $V_{0}$.

Le tableau 1 récapitule l'ensemble des données acquises.

\section{Discussion.}

Il apparaît que l'ensemble des modifications histologiques et biochimiques qui accompagnent la spécialisation de la cellule dans la production massive de la soie est principalement sous-tendu par des variations quantitatives des espèces de mRNA présentes initialement. On observe notamment l'accumulation, à côté du mRNAF, d'une autre espèce de fonction inconnue. 
TABLEAU 1

Distribution des mRNA du stade $V_{0}$ dans les différentes classes d'abondance des mRNA du stade $V_{6}$

\begin{tabular}{|c|c|c|c|}
\hline \multicolumn{2}{|c|}{ mRNA $V_{0}$} & \multicolumn{2}{|c|}{ mRNA $V_{6}$} \\
\hline $\begin{array}{c}\text { Classes } \\
\text { d'abondance }\end{array}$ & $\begin{array}{c}\text { Nombre } \\
\text { de séquences }\end{array}$ & $\begin{array}{c}\text { Nombre } \\
\text { de séquences }\end{array}$ & $\begin{array}{c}\text { Classes } \\
\text { d'abondance } \\
\text { (mRNAF-) }\end{array}$ \\
\hline
\end{tabular}

1 (mRNAF)

1

2

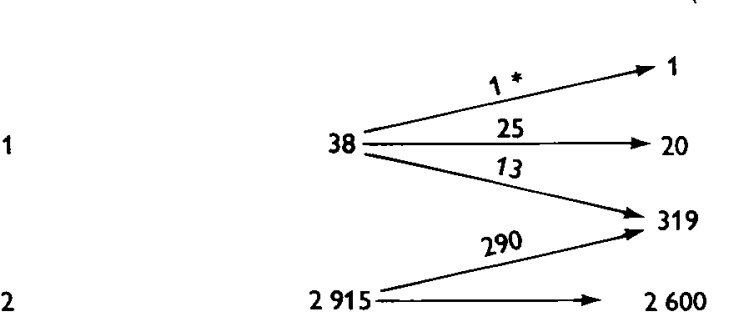

1

Ce tableau récapitule les données obtenues après exploitation des courbes présentées figure 4. Ainsi, le CDNA de la classe abondante du stade $V_{0}$, représentant 38 espèces de $m R N A$, s'hybride avec le mRNA F- du stade $V_{6}$ selon une cinétique définissant deux classes, estimées contenir respectivement 25 et 13 mRNA différents. De même l'hybridation hétérologue avec le cDNA de la classe rare du stade $V_{0}$ (2 915 mRNA) montre qu'il se répartit en deux classes de 291 et 2600 espèces. La correspondance entre mRNA des stades $V_{0}$ et $V_{6}$ est établie par comparaison des cinétiques d'hybridation homologue ef hétérologue.

* La présence de ce mRNA, particulièrement abondant au stade $V_{6}$, dans la classe des mRNA abondants au stade $V_{0}$, a été montrée en hybridant le mRNA $V_{0}$ avec le cDNA spécifique de cette espèce, purifié à partir du cDNA total $V_{6}$.

Ces modifications quantitatives aboutissent à une distribution plus hétérogène des mRNA, ce qui se traduit par l'augmentation du nombre de classes d'abondance. On peut supposer que les mRNA qui voient leur importance relative augmenter sont impliqués dans la production de fibroīne, alors que le petit nombre d'espèces initialement abondantes qui diminue relativement, pourraient être caractéristiques d'autres processus, ceux de la mue par exemple.

L'hypothèse de travail qui se dégage est que la différenciation terminale de la glande séricigène est essentiellement le résultat d'une modulation quantitative de l'expression des gènes. Le clonage de cDNA des diverses classes devrait fournir le moyen d'étudier le niveau - transcriptionnel ou postranscriptionnel — où cette modulation intervient.

6e Réunion du groupe Développement I.N.R.A., Clermont-Ferrand/Theix, 22-23 mai 1980.

Remerciements. - Ce travail a bénéficié d'un soutien financier du CNRS (ATP 3720) et de l'UER de Biologie Humaine de l'Université Claude Bernard. - Nous remercions le Docteur R. Axel pour ses suggestions ef le Docteur D. Kurtz qui nous a aimablement fourni la reverse transcriptase. 


\section{Références}

AVIV H., LEDER P., 1972. Purification of biologically active globin messenger RNA by chromatography on oligothymidilic acid cellulose. Proc. nat. Acad. Sci., U. S. A., 69, 1408-1412.

AXEL R., FEIGELSON P., SCHÜTZ G., 1976. Analysis of the complexity and diversity of mRNA from chicken liver and oviduct. Cell, 7, 247-254.

COUBLE P., GAREL A., PRUDHOMME J.-P., 1981. Complexity and diversity of polyadenylated mRNA in the silkgland of Bombyx mori. Changes related to fibroin production. Develop. Biol., 82 (sous presse).

GREENE R. A., MORGAN M., SHATKIN A. J., GAGE P., 1975. Translation of silk fibroin messenger RNA in an Ehrlich ascites cell-free extract. J. mol. Biol., 250, 5114-5121.

HASTIE N. D., BISHOP J. O., 1976. The expression of three abundance classes of messenger RNA in mouse tissues. Cell, 9, 761-774.

HYNES N. E., GRONER B., SIPPEL A. E., NGUYEN-HUU M. C., SCHUTZ G., 1977. mRNA complexity and egg white protein mRNA content in mature and hormone withdrawn oviduct. Cell, 11, 923-932.

KEMP D. J., 1975. Unique and repetitive sequences in multiple genes for feather keratin. Nature, 254, 573-576.

MORROW J. F., CHANG N. T., WOZNEY J. M., RICHARDS A. C., ESFRATIADIS A., 1977. In SCOTT W. A., WERNER R., Molecular cloning in recombinant DNA, 161-171, Acad. Press.

MYERS J. C., SPIEGELMAN S., 1978. Sodium pyrophosphate inhibition of RNA-DNA degradation by reverse transcriptase. Proc. nat. Acad. Sci., U. S. A., 75, 5329-5333.

PRUDHOMME J.-C., COUBLE P., 1979. The adaptation of the silkgland cell to the production of fibroin in Bombyx mori L. Biochimie, 61, 215-227.

SUZUKI Y., 1975. Fibroin messenger RNA and its gene. Adv. Biophys., 8, 83-114.

SUZUKI Y., 1977. Differentiation of the silkgland. A model system for the study of differential gene action, 1-44. In BEERMAN W., Biochemical differentiation in insects glands. Springer-Verlag, Berlin.

SUZUKI Y., BROWN D. D., 1972. Isolation and identification of the mRNA for silk fibroin from Bombyx mori. J. mol. Biol., 63, 409-429.

SUZUKI Y., SUZUKI E., 1974. Quantitative measurements of fibroin messenger RNA synthesis in the posterior silkgland of normal and mutant Bombyx mori. J. mol. Biol., 88, 393-407.

TASHIRO Y., MORIMOTO T., MATSUURA S., NAGATA S., 1968. Studies of the posterior silkgland of the silkworm Bombyx mori. J. Cell Biol., 38, 574-588. 